Article

\title{
Evaluation of a mesoscale thermal actuator in open and closed operating cycles
}

\author{
Sindhu Preetham Burugupally \\ Department of Mechanical Engineering, Wichita State University, Wichita, KS 67260, USA \\ * Correspondence: sindhupreetham.burugupally@wichita.edu
}

\begin{abstract}
Thermal-based actuators are known for generating large force and displacement strokes at mesoscale (millimeter) regime. In particular, two-phase thermal actuators are found to benefit from the scaling laws of physics at mesoscale to offer large force and displacement strokes; but they have low thermal efficiencies. As an alternative, a combustion-based thermal actuator is proposed and its performance is studied in both open and closed cycle operations. Through a physics-based lumped-parameter model, we investigate the behavior and performance of the actuator using a spring-mass-damper analogy and taking an air standard cycle approach. Three observations are reported: (1) the mesoscale actuator can generate peak forces of up to $400 \mathrm{~N}$ and displacement strokes of about $16 \mathrm{~cm}$ suitable for practical applications; (2) an increase in heat input to the actuator results in increasing the thermal efficiency of the actuator for both open and closed cycles; and (3) for a specific heat input, both the open and closed cycle operations respond differently —different stroke lengths, peak pressures, and thermal efficiencies.
\end{abstract}

Keywords: Thermal actuator, compliant architecture, open and closed operating cycles, mesoscale

\section{Introduction}

Mechanical actuation via thermal actuators has been a topic of interest due to their ability to generate forces $O\left(10^{1}\right) \mathrm{N}$ and displacement strokes $O\left(10^{-4}\right) \mathrm{m}$ at mesoscale (millimeter) regime [1]. Most thermal actuators employ either bimorph [2], solid expansion [3], fluid expansion [4], state change (liquid-to-vapor) [5], gas expansion/compression [6], or combustion based mechanisms [7]. Among them, state change and combustion based mechanisms are found to be suitable for large displacement and force generation at mesoscale as they benefit from the scaling laws of physics $[5,7]$. In particular, combustion-based miniature heat engines offer high power density to weight ratio and fast response, thus making ideal for applications that require high speed, large force, and large displacement strokes. These actuators have applications in exoskeleton [8], locomotion [9], and rehabilitation [10].

Unfortunately, thermal actuators or heat engines with sliding-piston architecture at mesoscale suffer from high friction, leakage, and heat losses, resulting in poor thermal efficiencies [11,12]. A method to improve the thermal efficiency is by eliminating the crankshaft assembly and associated friction losses [7]; this can be accomplished by implementing a flexible or compliant architecture (Fig. 1) instead of a sliding-piston architecture, which also offer other advantages - no leakage losses, simpler construction, and lower fabrication costs $[13,14]$.

Research on the fundamental design of thermal actuators (heat addition/rejection cycle [7]) or heat engines (Otto cycle) $[13,14]$ that employ compliant architecture are being studied through models [14], prototype fabrication and experiments [13]. First-principle based mathematical models are being developed to assess the performance of the compliant actuators/engines for a range of physical and operating parameters in both open and closed cycle operations. However, it is unclear whether such a thermal actuator/heat engine be analyzed or designed to operate in an open or a closed cycle. 
(a)

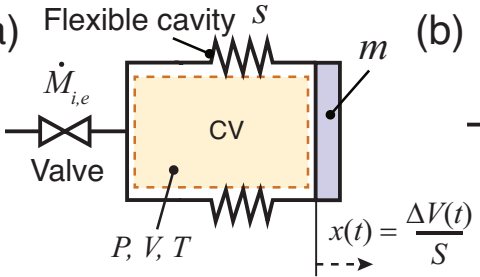

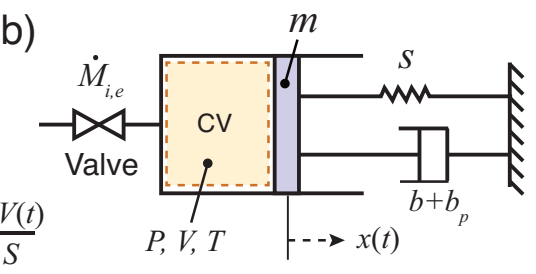

Figure 1. Proposed thermal actuator (a) schematic sketch and (b) lumped model. CV denotes the cavity or control volume with state parameters: pressure $P$, volume $V$, and temperature $T$ at time $t$. The valve on the left is used for the intake or exhaust of the working fluid (air). The valve is connected to the ambient atmosphere $\left(P_{o}, T_{0}\right)$ during the intake and exhaust processes. For the closed cycle, the valve remains closed over the entire operating cycle.

Our present work on thermal actuators builds on our prior physics-based modeling efforts of a resonant heat engine for portable power applications $[7,13,14]$. In this study, through a physics-based lumped-parameter model, similar to our previously developed models on free piston engines [15-17], we will investigate the behavior and performance of a mesoscale thermal actuator using a spring-mass-damper analogy and taking an air standard cycle approach. Here, using the model, we will predict the actuator performance for a range of operating conditions and report on the important differences between the open and closed cycle operations.

\section{Thermal Actuator: Design and Model}

The proposed actuator is similar to a traditional internal combustion engine, where fuel-air mixture is burnt in a piston-cylinder arrangement to produce force and displacement strokes. The actuator comprises of a flexible cavity with a piston attached at one end (Fig. 1). The flexible or compliant architecture reduces the frictional losses by up to $5 \%[13,14]$. The actuator can be operated in both open and closed cycles. The actuator when operated in the open cycle comprises of compression $(1 \rightarrow 2)$, heat addition $(2 \rightarrow 3)$, expansion $(3 \rightarrow 4)$, exhaust $(4 \rightarrow 5 \rightarrow 6)$, and intake $(6 \rightarrow 7 \rightarrow 1)$ processes as depicted in Fig. 2. Unlike the open cycle, the closed cycle terminates with the heat rejection process $(4 \rightarrow 1)$. In the open cycle, the actuator exchanges both heat and mass with the surroundings, while in the closed cycle only exchange of heat takes place.

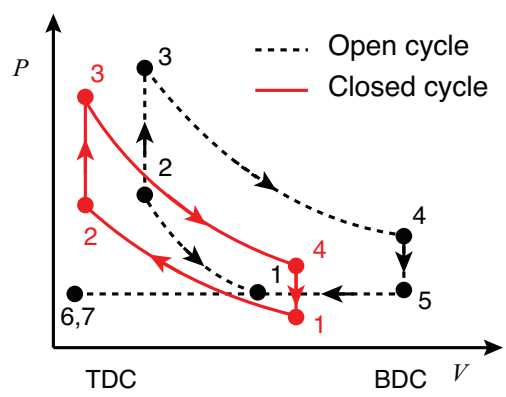

Figure 2. Ideal pressure-volume diagram of the actuator in open and closed cycle operations. Processes $1 \rightarrow 2$ : Compression; $2 \rightarrow 3$ : Heat addition for duration $t_{q}$; and $3 \rightarrow 4$ : Expansion. For closed cycle, process $4 \rightarrow 1$ is heat rejection for duration $t_{q}$. For open cycle, processes $4 \rightarrow 5$ : Blowdown phase of exhaust; $5 \rightarrow 6$ : Displacement phase of exhaust; $6 \rightarrow 7 \rightarrow 1$ : Intake. Note the piston positions at states 6 and 7 are 'Top Dead Center' (TDC) and states 4 and 5 are 'Bottom Dead Center' (BDC), respectively.

A physics-based model is derived to investigate the actuator behavior and performance for both the open and closed cycle operations. The actuator is modeled as a spring-mass-damper system by taking an air standard cycle approach [21] (Fig. 1b). The hollow cavity represents the control volume $\mathrm{CV}$ and contains the working fluid (air). The combustion process is modeled as a constant volume heat addition process to $\mathrm{CV}$ for a short duration $t_{q} \rightarrow 0$ and the heat rejection at the end of the expansion 
58

8

process is modeled as a mass transfer process (for open cycle) or by applying a cooling pulse (for closed cycle) for a short duration $t_{q} \rightarrow 0$ (Fig. 3). The compliant cavity CV has a nominal volume $V_{o}$ given by $V_{o}=L S$, where $L$ is the nominal cavity length and $S$ is the cross-sectional area of the cavity. The cavity has a stiffness $s$ which allows horizontal displacement $x(t)$ of the piston $m$. Damper $b$ models energy conversion which is the sum of both friction work and useful work by the actuator, and $b_{p}(<0)$ models work done on the actuator during the intake process. Over an operating cycle, the working fluid temperature, $T_{0}+\Delta T(t)$, pressure, $P_{o}+\Delta P(t)$, and cavity volume, $V_{o}+\Delta V(t)$ undergo cyclic variation, where 'o' subscript represents nominal or ambient conditions, while $\Delta$ represents time-dependent excursion.

The actuator model Eqs. [1-5] is derived by employing Newton's second law, conservation of mass, conservation of energy, an ideal gas model, and a linear mass flow-rate equation.

$$
\begin{gathered}
\frac{m}{S^{2}} \Delta \ddot{V}+\frac{b+b_{p}}{S^{2}} \Delta \dot{V}+\frac{s}{S^{2}} \Delta V=\Delta P \\
\dot{M}=\dot{M}_{i}-\dot{M}_{e} \\
h \Delta T+\frac{\mathrm{d}\left(M c_{v} T\right)}{\mathrm{d} t}+P \Delta \dot{V}=q+\dot{M}_{i} c_{p} T_{i}-\dot{M}_{e} c_{p} T \\
P V=M R T \\
\dot{M}_{i, e}=-k \Delta P
\end{gathered}
$$

where $M$ is the working fluid (air) mass, $h$ is the total heat loss coefficient, $R$ is the mass-specific gas constant of air, $k$ is the mass flow rate coefficient, and $c_{v}$ and $c_{p}$ are the constant volume and constant pressure heat capacities of air. The overdot denotes time derivative, while the subscripts ' $i$ ' and ' $e$ ' denote intake and exhaust processes, respectively. The model Eqs. [1-5] is nondimensionalized using the scaling terms given in Eq. 6 and then written in a state space format as shown in Eqs. [7-10].

$$
\begin{gathered}
\Delta \bar{V}=\frac{\Delta V}{V_{o}}, \Delta \bar{P}=\frac{\Delta P}{P_{o}}, \Delta \bar{T}=\frac{\Delta T}{T_{o}}, \bar{q}=\frac{q}{q_{H}}, \text { and } \bar{t}=t \omega \\
\frac{d \Delta \bar{V}}{d \bar{t}}=\Delta \dot{\bar{V}} \\
\frac{d \Delta \dot{\bar{V}}}{d \bar{t}}=\frac{\Delta \bar{P}}{\gamma}-\frac{b+b_{p}}{m \omega} \Delta \dot{\bar{V}}-\frac{s}{m \omega^{2}} \Delta \bar{V} \\
\Delta \dot{\bar{T}}=\frac{1}{M c_{v} T_{o} \omega}\left[q+\dot{M}_{i} T_{o}\left[R+c_{p} \Delta \bar{T}_{i}-c_{v} \Delta \bar{T}\right]-h T_{o} \Delta \bar{T}-\dot{M}_{e} R T_{o}(1+\Delta \bar{T})-P_{o} V_{o} \omega(1+\Delta \bar{P}) \Delta \dot{\bar{V}}\right] \\
\Delta \dot{\bar{P}}=\frac{1}{P_{o} V_{o} \omega(1+\Delta \bar{V})}\left[\left(\dot{M}_{i}-\dot{M}_{e}\right) R T_{o}(1+\Delta \bar{T})+M R T_{o} \omega \Delta \dot{\bar{T}}-P_{o} V_{o} \omega(1+\Delta \bar{P}) \Delta \dot{V}\right] \\
\text { where } \omega=\sqrt{\frac{\gamma P_{o} S^{2}}{m V_{o}}} \text { is a reference frequency and the overbar denotes a nondimensional term. }
\end{gathered}
$$



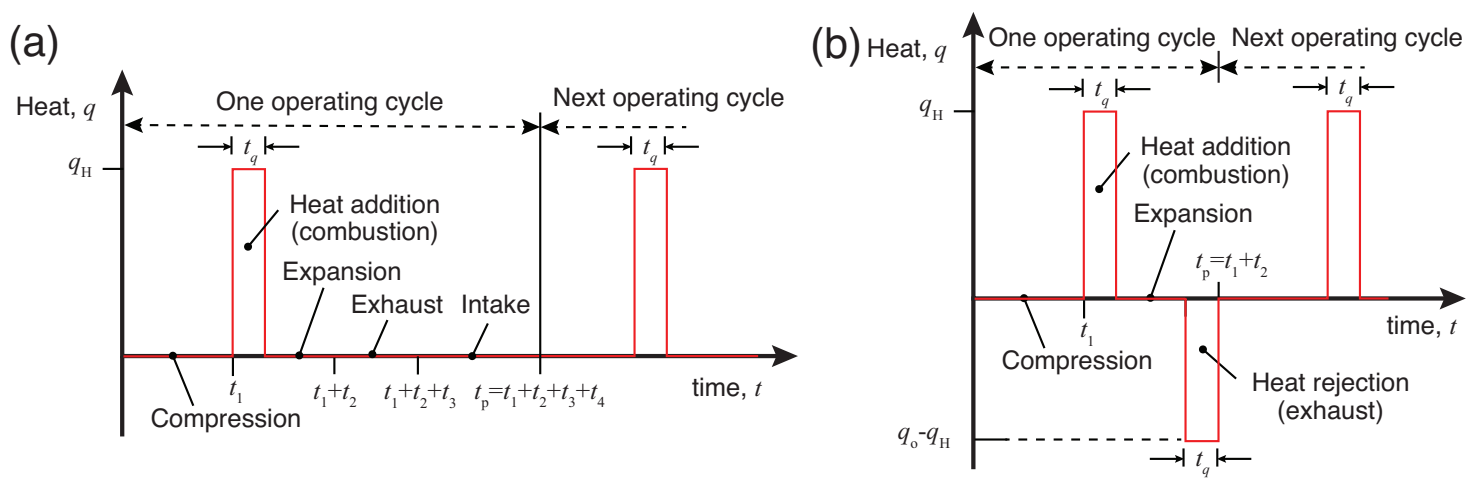

Figure 3. Heat-rate function $q$ for modeling the (a) open and (b) closed operating cycles.

\subsection{Solving the open cycle model}

To generate a pressure-volume diagram, the model Eqs. [7-10] is numerically integrated in the order $1 \rightarrow 2 \rightarrow 3 \rightarrow 4 \rightarrow 5 \rightarrow 1$ (Fig. 2) starting with state $1: \Delta \bar{V}_{1}, \Delta \bar{P}_{1}, \Delta \bar{T}_{1}, \Delta \dot{V}_{1}=0$. The resulting volume $\Delta \bar{V}_{2}$, pressure $\Delta \bar{P}_{2}$, and temperature $\Delta \bar{T}_{2}$ from the compression process, $1 \rightarrow 2$ is computed by integrating Eqs. [7-10] from the initial condition $\Delta \bar{V}_{1}, \Delta \bar{P}_{1}, \Delta \bar{T}_{1}, \Delta \dot{V}_{1}=0$ for a time $\bar{t}_{1}$ such that the piston velocity $\dot{V}_{2}=0$. The resulting volume $\Delta \bar{V}_{3}$, pressure $\Delta \bar{P}_{3}$, and temperature $\Delta \bar{T}_{3}$ during the heat addition process, $2 \rightarrow 3$ is computed from Eqs. 8 and 9 by assuming it to be instantaneous $\left(\Delta \bar{V}_{3}=\Delta \bar{V}_{2}\right.$ and $\left.\Delta \dot{V}_{3}=\Delta \dot{V}_{2}\right)$. The volumes, pressures, and temperatures $\Delta \bar{V}_{4}, \Delta \bar{P}_{4}, \Delta \bar{T}_{4} ; \Delta \bar{V}_{6}, \Delta \bar{P}_{6}, \Delta \bar{T}_{6}$; and $\Delta \bar{V}_{1}, \Delta \bar{P}_{1}, \Delta \bar{T}_{1}$ from the expansion $3 \rightarrow 4$, exhaust (displacement phase) $5 \rightarrow 6$, and intake processes $6 \rightarrow 7 \rightarrow 1$ are computed by integrating Eqs. [7-10] from the initial conditions $\Delta \bar{V}_{3}, \Delta \bar{P}_{3}, \Delta \bar{T}_{3}, \Delta \dot{V}_{3}=0$; $\Delta \bar{V}_{5}, \Delta \bar{P}_{5}, \Delta \bar{T}_{5}, \Delta \bar{V}_{5}=0$; and $\Delta \bar{V}_{6}, \Delta \bar{P}_{6}, \Delta \bar{T}_{6}, \Delta \dot{V}_{6}=0$ for times $\bar{t}_{2}, \bar{t}_{3}$, and $\bar{t}_{4}$ respectively such that the piston velocity $\Delta \dot{\bar{V}}_{4}=\Delta \dot{\bar{V}}_{5}=0$ and $\Delta \dot{\bar{V}}_{6}=\Delta \dot{\bar{V}}_{7}=0$. The times $\bar{t}_{1}, \bar{t}_{2}, \bar{t}_{3}$, and $\bar{t}_{4}$ are unknowns and are determined during the process of integration. The blowdown process $4 \rightarrow 5$ is assumed instantaneous, isentropic, and occurs at zero piston velocity $\left(\Delta \dot{\bar{V}}_{4}=\Delta \dot{\bar{V}}_{5}=0\right)$ and ambient pressure $\left(\Delta \bar{P}_{5}=0\right)$. The working fluid temperature at state $5, \Delta \bar{T}_{5}$ is determined using the adiabatic relation:

$$
\Delta \bar{T}_{5}=\left(1+\Delta \bar{T}_{4}\right)\left(\frac{1+\Delta \bar{P}_{4}}{1+\Delta \bar{P}_{5}}\right)^{\frac{1-\gamma}{\gamma}}-1
$$

\subsection{Solving the closed cycle model}

To generate a pressure-volume diagram, the model Eqs. [7-10] is numerically integrated in the order $1 \rightarrow 2 \rightarrow 3 \rightarrow 4 \rightarrow 1$ (Fig. 2) starting with state $1: \Delta \bar{V}_{1}, \Delta \bar{P}_{1}, \Delta \bar{T}_{1}, \Delta \dot{\bar{V}}_{1}=0$. The resulting volume $\Delta \bar{V}_{2}$, pressure $\Delta \bar{P}_{2}$, and temperature $\Delta \bar{T}_{2}$ from the compression process, $1 \rightarrow 2$ is computed by integrating Eqs. [7-10] from the initial condition $\Delta \bar{V}_{1}, \Delta \bar{P}_{1}, \Delta \bar{T}_{1}, \Delta \dot{V}_{1}=0$ for a time $\bar{t}_{1}$ such that the piston velocity $\dot{\bar{V}}_{2}=0$. The resulting volume $\Delta \bar{V}_{3}$, pressure $\Delta \bar{P}_{3}$, and temperature $\Delta \bar{T}_{3}$ during the heat addition process $(2 \rightarrow 3)$ is computed from equations 8 and 9 by assuming it to be instantaneous $\left(\Delta \bar{V}_{3}=\Delta \bar{V}_{2}\right.$ and $\Delta \dot{\overline{V_{3}}}=\Delta \dot{\bar{V}}_{2}$ ). The volume, pressure, and temperature $\Delta \bar{V}_{4}, \Delta \bar{P}_{4}, \Delta \bar{T}_{4}$ from the expansion process $3 \rightarrow 4$ is computed by integrating Eqs. [7-10] from the initial conditions $\Delta \bar{V}_{3}, \Delta \bar{P}_{3}, \Delta \bar{T}_{3}, \Delta \dot{V}_{3}=0$ for time $\bar{t}_{2}$ such that the piston velocity $\Delta \dot{\bar{V}}_{4}=\Delta \dot{\bar{V}}_{1}=0$. The times $\bar{t}_{1}$ and $\bar{t}_{2}$ are unknowns and are determined during the process of integration. The heat rejection process $4 \rightarrow 1$ is assumed to be instantaneous and occurs at zero piston velocity $\left(\Delta \dot{\bar{V}}_{4}=\Delta \dot{\bar{V}}_{1}=0\right)$.

\section{Results and Discussion}

The actuator with $h=0 \mathrm{~W} / \mathrm{K}, b=21.5 \mathrm{~N}-\mathrm{s} / \mathrm{m}, k=6.8 \times 10^{-7} \mathrm{~kg} / \mathrm{s}-\mathrm{Pa} \pm 20 \%, V_{o}=21.8 \mathrm{cc}, s=3050 \mathrm{~N} / \mathrm{m}$, $m=0.18 \mathrm{~kg}$, and clearance volume $V_{c}=0.01 V_{o}$ are treated as the reference. These numerical values are experimentally determined [13]. $T_{o}=295 \mathrm{~K}$ and $P_{o}=93.4 \mathrm{kPa}$ are the ambient and standard conditions. 

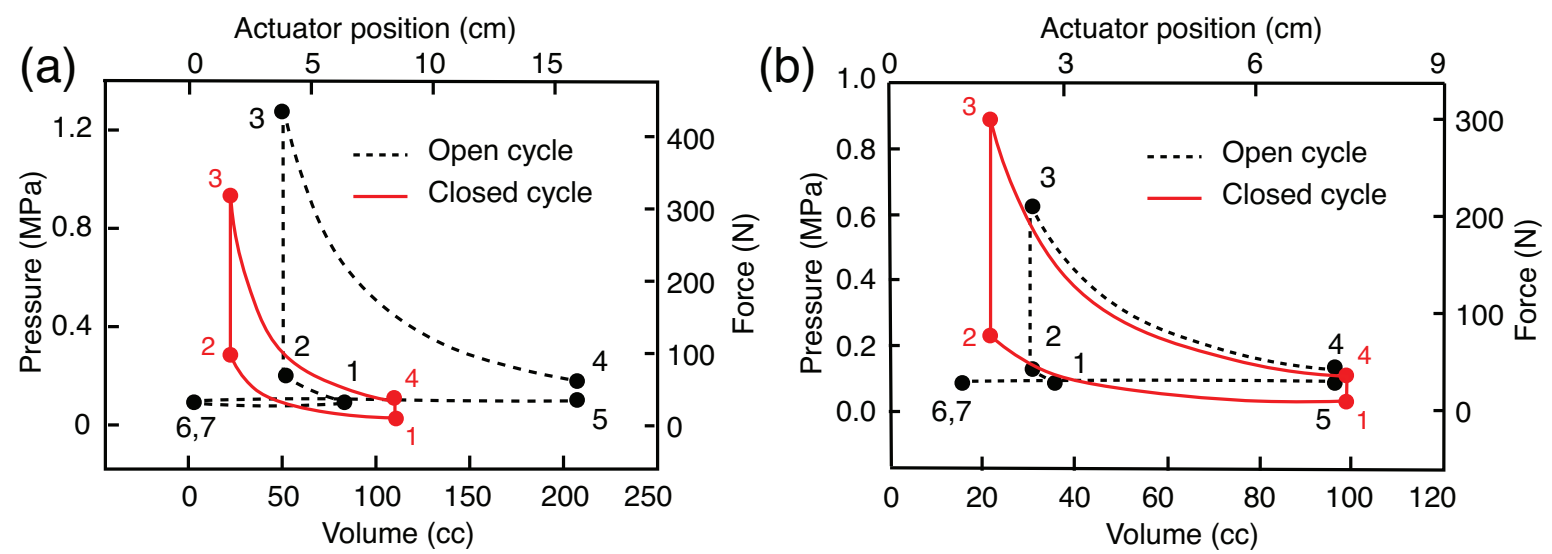

Figure 4. Open and closed cycle pressure-volume and force-displacement diagrams for (a) the reference actuator (b) an actuator with $b=27.5 \mathrm{~N}-\mathrm{s} / \mathrm{m}, b_{p}=-40 \mathrm{~N}-\mathrm{s} / \mathrm{m}$ and $\phi=0.51$.

\subsection{Pressure-Volume and Force-Displacement diagrams}

The PV diagrams for both the open and closed cycles shown in Fig. 4 are generated by applying a heat pulse that corresponds to lean combustion (equivalence ratio $\phi=0.51$ [20]) of octane in the actuator (Fig. 3). The peak pressure $P_{3}$ in the actuator for the open and closed cycles are off by a factor of 1.3 (Fig. 4a). Note that the temperature rise in both the cycles are equal $\left(\Delta T_{23}=2064 \mathrm{~K}\right)$ because the equivalence ratio for both the cycles are equal $(\phi=0.51)$. Also, note that the pressure rise $\Delta P_{23}$ among the cycles is different, as the density of air at the thermodynamic state 2 in both the cycles are unequal -for the closed cycle, the density at state 2 is $1.10 \mathrm{~kg} / \mathrm{m}^{3}$, while for the open cycle, the density at state 2 is $1.87 \mathrm{~kg} / \mathrm{m}^{3}$. The actuator pressure at the end of heat rejection or blow down process is ambient pressure $\left(P_{5}=0 \mathrm{kPa}\right.$ gage $)$ and below the ambient pressure $\left(P_{5}=-11 \mathrm{kPa}\right.$ gage $)$ for the open and closed cycles, respectively. This is because the open cycle interacts with the surroundings via mass transfer that allows the actuator pressure to quickly equilibrate to the ambient conditions, while in the closed cycle there is no mechanism, that is, no mass transfer or heat transfer $h=0 \mathrm{~W} / \mathrm{K}$ to equilibrate the actuator pressure to the ambient conditions.

In the context of force and displacement generated, the actuator produces a peak force of up to $400 \mathrm{~N}$ and displacement stroke of up to $16 \mathrm{~cm}$ (Fig. $4 \mathrm{a}$ ). Note that the force generated is not uniform over the displacement range, but reduces in magnitude with the displacement.

Table 1. Comparison of the performance metrics for the reference actuator in closed and open cycles with equivalence ratio $\phi=0.51$.

\begin{tabular}{lcc}
\hline Performance Parameter & Closed cycle & Open cycle \\
\hline Indicated thermal efficiency (\%) & 47.66 & 38.41 \\
Brake thermal efficiency (\%) & 44.33 & 34.64 \\
Heat added per cycle (J/cycle) & 35.6 & 138.5 \\
Cycle frequency (Hz) & 37.03 & 13.37 \\
Compression ratio, CR & 5.05 & 1.76 \\
Expansion ratio, ER & 5.05 & 4.13 \\
Average piston speed (m/s) & 4.75 & 4.77 \\
Power output (W) & 584.7 & 641.6 \\
Mass of air in cylinder (kg/cycle) & $2.41 \times 10^{-5}$ & $9.28 \times 10^{-5}$
\end{tabular}

The work done by the reference actuator in both the closed and open cycles for $\phi=0.51$ are found to be $16.96 \mathrm{~J}$ and $53.19 \mathrm{~J}$. These correspond to indicated thermal efficiencies of $47.66 \%$ and $38.41 \%$, 
respectively. The CR and ER for the closed cycle are equal to 5.05, while the CR and ER for the open cycle are found to be 1.76 and 4.13 , respectively (Table 1 ). This result indicates that despite the same equivalence ratio $\phi$ the open and closed cycles respond differently with different performance metrics quantified in terms of stroke lengths, peak pressures, and indicated work.

For the reference actuator, the addition of extra load (an increment by $1.5 \mathrm{~N}-\mathrm{s} / \mathrm{m}$ ), results in shorter displacement strokes, and the addition of external work modeled by $b_{p}=-40 \mathrm{~N}-\mathrm{s} / \mathrm{m} \mathrm{during}$ the intake process $7 \rightarrow 1$ results in significantly altering the PV diagram for the open cycle (comparing Fig. $4 \mathrm{a}$ and $4 \mathrm{~b}$ ). The actuator performance metrics for the open and closed cycles are given in Table 1.

\subsection{Equivalence ratio and heat input relationship}

For a specific fuel, an equivalence ratio is dependent on the mass of fuel injected (or associated heat input) and the mass of air inducted in to the actuator [21]. Fig. 5 graphically shows the relation between heat input and corresponding equivalence ratio $\phi$ for the reference actuator in open and closed cycles. Each plot in the figure is obtained by supplying the actuator with varying heat, and the corresponding equivalence ratios are calculated based on the mass of air in the cavity CV at state 1. It is clear from the figure that for a fixed equivalence ratio, the amount of heat added per cycle is higher for open cycle operation compared to a closed cycle operation. Furthermore, from the figure, the closed cycle operation holds a linear relationship between the equivalence ratio and heat input, while the open cycle holds a nonlinear linear for low $\phi(<0.23)$ and eventually transmutes to linear higher $\phi(>0.23)$. A similar observation has been reported in Ref. [14].

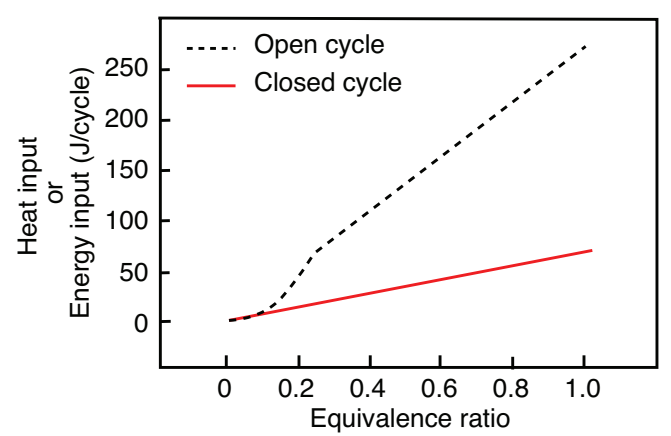

Figure 5. Relationship between the heat input and equivalence ratio for the reference actuator for open and closed cycle operations.

\subsection{Efficiency and volume ratios}

For a fixed equivalence ratio (say $\phi=0.5$ ), a difference of about $10 \%$ in indicated thermal efficiency is observed between the open and closed cycles (Fig. 6). The higher efficiencies of the closed cycle operations are because of ignoring the pumping losses in the actuator associated with the exhaust and intake processes. Furthermore, as can be seen in figure, an increase in the heat input or equivalence ratio $\phi$ increases the volume ratio (ER or CR). This is because a higher heat input or equivalence ratio $\phi$ will result in the higher pressure at the thermodynamic state 3 (Fig. 4), resulting in pushing the piston further outward and causing higher volume ratios.

\subsection{Operating frequency and power output}

It is evident from Fig. 7 that the operating frequency and output power from the actuator depends on the equivalence ratio. For the closed cycle, the operating frequency decreases monotonically with increasing equivalence ratio. On the contrary, for the open cycle, the operating frequency first decreases and later increases. This is because the actuator at state 6 (Fig. 2) reaches its lower displacement limit with a nonzero piston velocity - resulting in an abrupt changes the direction of 


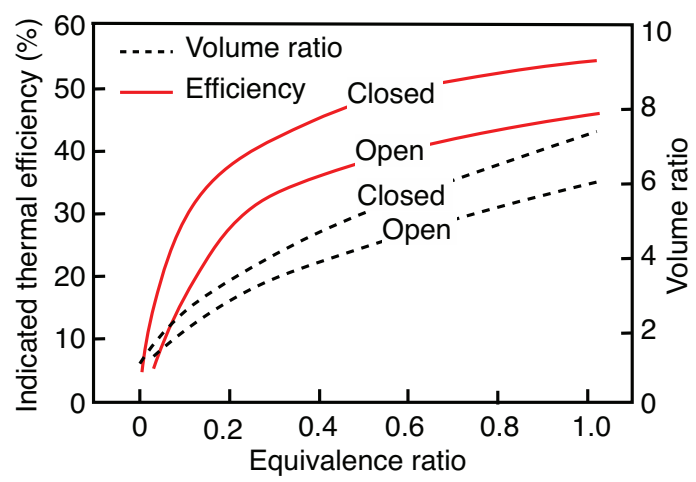

Figure 6. Efficiency and volume ratios for the reference actuator for open and closed cycles.

actuator motion-reducing the travel distance and time period or increasing the operating frequency of the operating cycle. For the reference actuator, the operating frequency for a closed cycle is $37.03 \mathrm{~Hz}$ while for an open cycle is $13.37 \mathrm{~Hz}$. The operating frequency depends predominantly on the number of strokes involved in an operating cycle, higher the number of strokes lower is operating frequency. For instance, a closed cycle has two strokes, while an open cycle has four strokes.

(a)

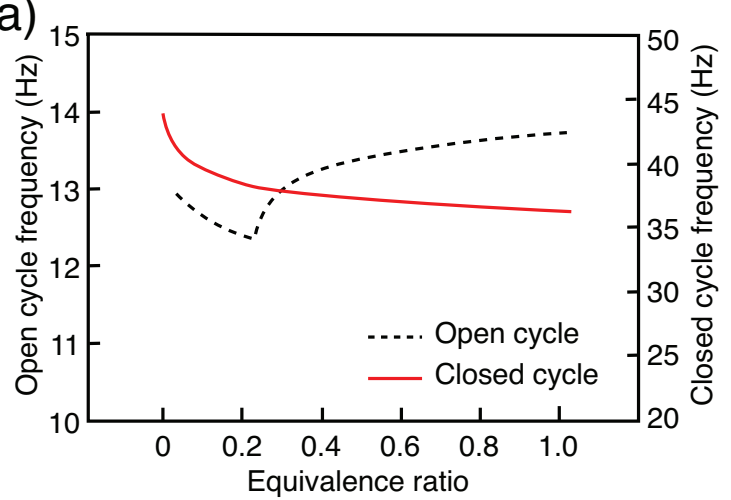

(b)

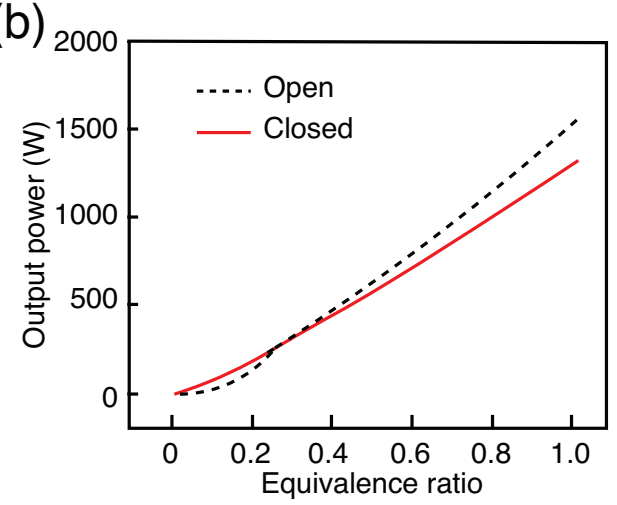

Figure 7. Performance metrics of the reference actuator in open and closed operating cycles: (a) Operating frequency and (b) Power output.

In the context of power output, the actuator produces a peak output power of up to $1500 \mathrm{~W}$ for an equivalence ratio of $\phi=1$ (Fig. $7 \mathrm{~b}$ ). The power output is a product of energy output and operating frequency of the actuator. Despite the operating frequency moderately increasing or decreasing, the trend in output power is fairly increasing - because the efficiency of the actuator and the energy input or output increases with the equivalence ratio $\phi$ (Figs. 5 and 6).

\section{Conclusions}

This work describes the difference in the behavior and performance of a mesoscale thermal actuator in both open and closed operating cycles. For this, a physics-based lumped parameter model of the actuator is derived using a spring-mass-damper analogy and taking an air standard cycle approach. Three observations are reported: (1) the mesoscale actuator can generate peak forces of up to $400 \mathrm{~N}$ and displacement strokes of about $16 \mathrm{~cm}$ suitable for practical applications; (2) an increase in heat input to the actuator results in increasing the thermal efficiency of both open and closed cycles; and (3) for a specific heat input, both the open and closed cycle operations respond differently - different stroke lengths, peak pressures, and thermal efficiencies.

Conflicts of Interest: The author declares no conflict of interest.

\section{Abbreviations}


The following abbreviations are used in this manuscript:

ER Expansion Ratio

CR Compression Ratio

CV Control Volume or Cavity

TDC Top Dead Center

BDC Bottom Dead Center

\section{References}

1. Bell, D.J.; Lu, T.J.; Fleck, N.A.; Spearing, S.M. MEMS actuators and sensors: observations on their performance and selection for purpose. Journal of Micromechanics and Microengineering 2005, 15, S153-S164. doi:10.1088/0960-1317/15/7/022.

2. Henriksson, J.; Gullo, M.; Brugger, J. Integrated long-range thermal bimorph actuators for parallelizable Bio-AFM applications. SENSORS, 2012 IEEE, 2012, pp. 1-4. doi:10.1109/ICSENS.2012.6411507.

3. Butler, J.T.; Bright, V.M.; Cowan, W.D. Average power control and positioning of polysilicon thermal actuators. Sensors and Actuators A: Physical 1999, 72, 88 - 97. doi:https:/ /doi.org/10.1016/S0924-4247(98)00211-8.

4. Lu, T.; Hutchinson, J.; Evans, A. Optimal design of a flexural actuator. Journal of the Mechanics and Physics of Solids 2001, 49, 2071 - 2093. The JW Hutchinson and JR Rice 60th Anniversary Issue, doi:https:/ /doi.org/10.1016/S0022-5096(01)00024-2.

5. Bardaweel, H.; Preetham, B.S.; Richards, R.; Richards, C.; Anderson, M. MEMS-based resonant heat engine: scaling analysis. Microsystem Technologies 2011, 17, 1251-1261. doi:10.1007/s00542-011-1306-y.

6. Nakajima, N.; Ogawa, K.; Fujimasa, I. Study on micro engines-miniaturizing Stirling engines for actuators and heatpumps. IEEE Micro Electro Mechanical Systems, , Proceedings, 'An Investigation of Micro Structures, Sensors, Actuators, Machines and Robots', 1989, pp. 145-148. doi:10.1109/MEMSYS.1989.77979.

7. Preetham, B.S.; Anderson, M.; Richards, C. Modeling of a resonant heat engine. Journal of Applied Physics 2012, 112, 124903, [https:/ / doi.org/10.1063/1.4769447]. doi:10.1063/1.4769447.

8. Ouyang, X.; Ding, S.; Fan, B.; Li, P.Y.; Yang, H. Development of a novel compact hydraulic power unit for the exoskeleton robot. Mechatronics 2016, 38, 68 - 75. doi:https://doi.org/10.1016/j.mechatronics.2016.06.003.

9. Raibert, M.; Blankespoor, K.; Nelson, G.; Playter, R. Bigdog, the rough-terrain quadruped robot. IFAC Proceedings Volumes 2008, 41, 10822-10825.

10. Bradley, D.; Acosta-Marquez, C.; Hawley, M.; Brownsell, S.; Enderby, P.; Mawson, S. NeXOS - The design, development and evaluation of a rehabilitation system for the lower limbs. Mechatronics 2009, 19, 247 257. doi:https://doi.org/10.1016/j.mechatronics.2008.07.006.

11. Formosa, F.; Fréchette, L.G. Scaling laws for free piston Stirling engine design: Benefits and challenges of miniaturization. Energy 2013, 57, 796 - 808. doi:https:/ / doi.org/10.1016/j.energy.2013.05.009.

12. Burugupally, S.P.; Weiss, L. Power Generation via Small Length Scale Thermo-Mechanical Systems: Current Status and Challenges, a Review. Energies 2018, 11. doi:10.3390/en11092253.

13. Preetham, B.S.; Anderson, M.; Richards, C. Estimation of parasitic losses in a proposed mesoscale resonant engine: Experiment and model. Journal of Applied Physics 2014, 115, 054904, [https:/ / doi.org/10.1063/1.4864418]. doi:10.1063/1.4864418.

14. Preetham, B.S.; Anderson, M.; Richards, C. Mathematical modeling of a four-stroke resonant engine for micro and mesoscale applications. Journal of Applied Physics 2014, 116, 214904, [https:/ / doi.org/10.1063/1.4903217]. doi:10.1063/1.4903217.

15. Burugupally, S.P.; Weiss, L. Design and performance of a miniature free piston expander. Energy 2019, 170, 611 - 618. doi:https:/ / doi.org/10.1016/j.energy.2018.12.158.

16. Burugupally, S.P.; Weiss, L.; Depcik, C. The effect of working fluid properties on the performance of a miniature free piston expander for waste heat harvesting. Applied Thermal Engineering 2019, 151, $431-438$. doi:https:/ / doi.org/10.1016/j.applthermaleng.2019.02.035.

17. Preetham, B.; Weiss, L. Investigations of a new free piston expander engine cycle. Energy 2016, 106, 535 545. doi:10.1016/j.energy.2016.03.082.

18. Burugupally, S.; Weiss, L. Power generation via small length scale thermo-mechanical systems: current status and challenges, a review. Energies 2018, 11, 2253. 
227 19. Preetham, B.; Anderson, M.; Richards, C. Estimation of parasitic losses in a proposed mesoscale resonant 228 engine: Experiment and model. Journal of Applied Physics 2014, 115, 054904.

229 20. Heywood, J. Internal Combustion Engine Fundamentals; McGraw-Hill Education, 1988.

230 21. Pulkrabek, W. Engineering Fundamentals of the Internal Combustion Engine; Prentice Hall, 1997. 